\title{
9.3 Planetary cam mechanisms of one-directional movement with a stop
}

There is always a need for mechanisms, that makes it possible to accelerate and stop the driven links without shocks, so as ones designed to drive the links in one direction with a periodic stop [564]. Such planetary cam mechanisms has the rocker arm, which rotates around a fixed cam. All of planetary cam mechanisms presents in Fig. 1 and works on the same principle.

The scheme of the planetary cam-gear mechanism according to [565] is presented in Fig.1, a. The carrier 1 is mounted freely on the shaft 8, rotates counterclockwise at a constant speed. During rotation, the roller 5 of the rocker arm 4 rolls along a constant radius of the fixed groove cam 6 and drives the shaft 8 through the gear sector 2 and the gear wheel 7 at the same speed. The gear sector 2 and the rocker arm 4 are mounted on an axis pin 3, which rotates freely in the carrier 1 .

To stop the driven link 9 , the roller 5 passes to the variable radius of the cam profile 6 and rotates the gear 7 through the gear sector 2 in the opposite direction. If the angular velocity given to it by the toothed sector 2 is achieved, it stops and will stand until these angular velocities are equal. Rocker arm 4 reduces the angular velocity of the gear 7 to zero and accelerates it in the opposite direction at the end of the stop.

In the article [566] it is noted that obtaining a long stop of the driven link 9 requires a large angular span of the rocker arm of the cam mechanism. At the same time, the value of the angular span of the rocker arm should not exceed 60 degrees [564].

The proposed new planetary cam-gear mechanism with multiplier makes it possible to reduce significantly the swing angle of the rocker arm of the cam mechanism in order to increase the stopping time of the driven link. The mechanism works similarly to the previous one.

The multiplier of this mechanism (Fig. 1, b) consists of a gear wheel 10 mounted on a pin 3 , which rotates freely in the carrier 1 , and a gear sector 12 connected to the rocker arm 4 . They can swing on the finger 13, which hinged in the carrier 1. 

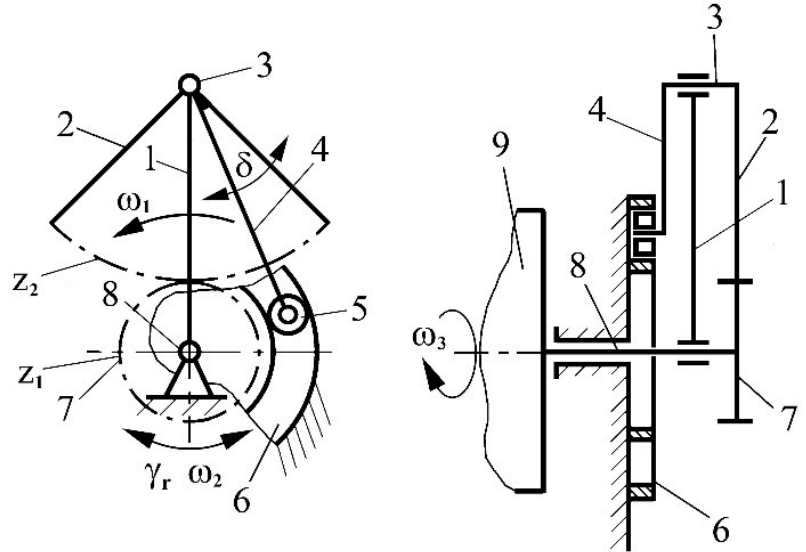

a)

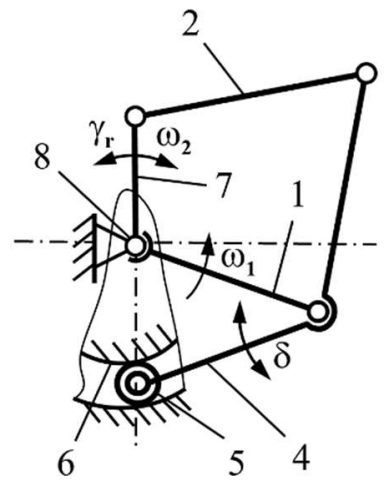

c)
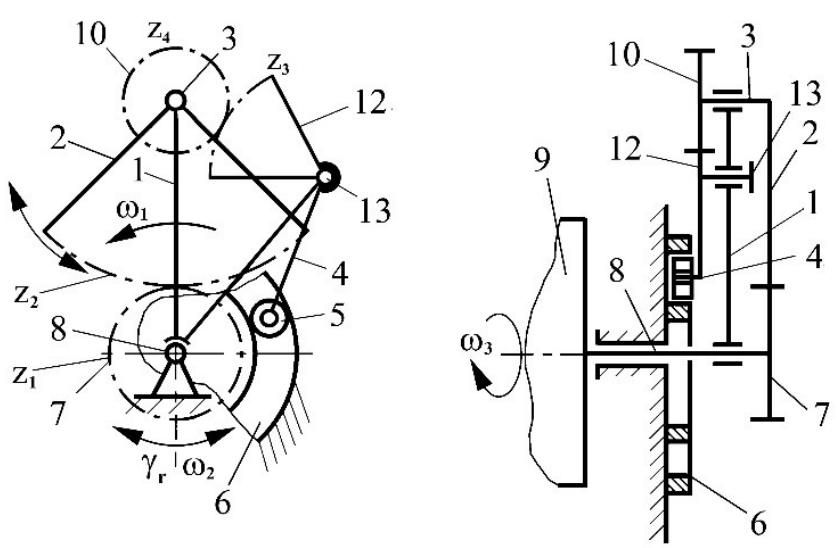

b)

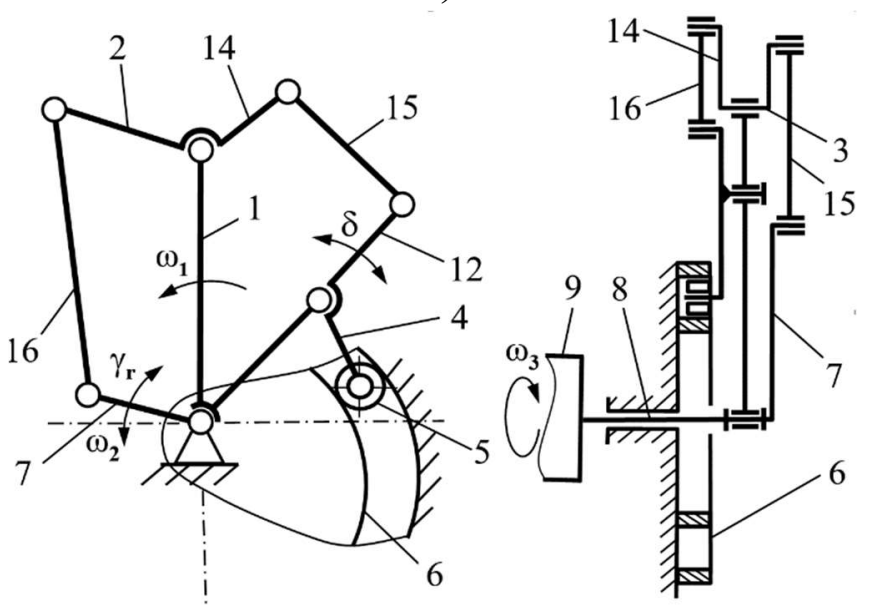

d)

Figure 1. Schemes of planetary cam mechanisms:

$a$ - cam-and-gear, $b$ - cam-and-gear with multiplier,

$c$ - cam-and-lever, $d$ - cam-and-lever with multiplier

On the other side of the carrier 1 on the axis pin 3 is fixed a gear sector 2, which interacts with the gear 7 connected to the shaft 8 . The gears 12 and 10 , as well as the gears 2 and 7 allows to increase significantly the angle of rotation of the gear 7 and to achieve a long stop of the driven link 9 .

From another point of view, the use of gears in these mechanisms (Fig. $1 \mathrm{a}, \mathrm{b}$ ) negatively affects the durability and accuracy of the mechanism. The reason is that they have gaps and contact occurs along the line. 
In order to eliminate these shortcomings, a cam-lever mechanism is proposed [567] (Fig. 1, c), where the movement of the two-arm rocker 4 is transmitted to the shaft 8 by the connecting rod 2 and the rocker arm 7 . During the rotation of the carrier 1 at a constant speed, the rocker arm 7 gives the shaft 8 the same speed in the opposite direction. As a result, the shaft 8 with the driven link 9 stops and stands still during the phase of equality of speeds. This mechanism, like the mechanism in Fig. 1, a, has restrictions on the relatively large phase angles of a stop of the driven link.

In order to eliminate the shortcomings inherent in gears, new planetary camlever mechanism is proposed (Fig. 1, d). In the mechanism, movement of the rocker arm 4 of the cam mechanism is transmitted to the shaft 8 by means of a multiplier, which consists of levers $12,15,14$, and then by a help of levers $2,16,7$. Thus, the mechanism extends the use by increasing the swing angle of the rocker arm 7 and, accordingly, the phase angle of driven link 9 stop, which ensures its longevity and better accuracy.

In packaging machines, planetary cam mechanisms can be used in the drive of conveyors to move the product from one position to the next with a stop to perform technological operations. For example, a similar mechanism (Fig. 1, a) used in die cutting press Autoplatina of Swiss company BOBST for stepwise movement of cardboard blanks to technological sections $[566,568]$.

In printing machines, the planetary cam-lever mechanism (Fig. 1, c) can be used to drive a rotating stop-gripper mechanism. It is designed to fix a motionless sheet on the register table, accelerate and transmit it to grippers of the printing cylinder, which rotates at a high constant angular velocity. This mechanism has high requirements for accuracy. He must take the sheet from the register table and accurately, without displacement, pass it to grippers of the printing cylinder.

Next, the authors presents the calculation method of the mechanisms' cycloramas, builds on the method of $[566,569]$.

The angular velocity $\omega_{3}$ of the shaft 8 and the driven link 9 consists of the carrying speed $\omega_{1}$ of the carrier 1 and the relative speed $\omega_{2}$, which gives it the link 7 
(gear Fig. 1 a, b, or rocker Fig. 1 c, d), the drive which is carried out from a fixed cam 6. This can be written as follows

$$
\omega_{3}=\omega_{1}+\omega_{2} .
$$

The angular velocity of the link 7 for the period of running up can be written as [570]:

$$
\omega_{2 \text { rup }}=\mathrm{b}_{\mathrm{k}_{1}} \cdot \frac{\gamma_{1}}{\varphi_{1}} \cdot \omega_{1}
$$

where $b_{k 1}$ is the speed invariant;

$\gamma_{1}$ is an angle of rotation of the link 7 during the running up period;

$\varphi_{1}$ is the phase angle of the running up period.

Similarly, the angular velocity of the link 7 is determined during the run-down period.

$$
\omega_{2 \mathrm{rd}}=\mathrm{b}_{\mathrm{k}_{3}} \cdot \frac{\gamma_{3}}{\varphi_{3}} \cdot \omega_{1}
$$

where index 3 denotes the same values as in formula (2), only for the run-down period.

For the stop of the shaft 8 it is necessary that the maximum speed provided by the link 7 is equal to the speed of the carrier 1, but has the opposite sign.

This condition can be written as follows:

$$
\omega_{3}=\omega_{2 \max }+\omega_{1}=0
$$

Here $\omega_{2 \max }=B_{1} \cdot \frac{\gamma_{1}}{\varphi_{1}} \cdot \omega_{1}-$ the maximum angular velocity of the link 7 , where $B_{1}$ is the peak constant of the selected law of motion of the link 7 during the run-up period.

Then it follows that

$$
\omega_{3}=B_{1} \cdot \frac{\gamma_{1}}{\varphi_{1}} \cdot \omega_{1}+\omega_{1}=0
$$


From here, we obtain absolute values of the phase angles of running up and down.

$$
\begin{aligned}
& \varphi_{1}=B_{1} \cdot \gamma_{1} ; \\
& \varphi_{3}=B_{3} \cdot \gamma_{3},
\end{aligned}
$$

where $B_{3}$ is the peak constant of the selected law of motion of the link 7 during the rundown period.

For a segment of the constant speed of the link 7 is determined similarly.

$$
\varphi_{2}=B_{2} \cdot \gamma_{2} ; \quad \varphi_{2}=\gamma_{2}
$$

where $B_{2}=1$ is the peak constant of a velocity during the uniform motion period.

Thus, the phase angle of rise of a follower will be equal to (Fig. 2):

$$
\varphi_{\mathrm{rs}}=\varphi_{1}+\varphi_{2}+\varphi_{3} .
$$

Next, the roller can roll on the profile of the cam with a constant maximum radius (a dwell), while the speed of the link 7 will be zero $\omega_{2}=0$. The phase angle of the section is denoted by $\varphi_{4}$ (fig. 2).

The presence the section is such in some cases, for example, when using this mechanism to drive the rotating stop-gripper mechanism in printing machines. It is necessary to ensure rotation of the stop-gripper at a constant speed $\omega_{1}$ during the transfer of the sheet of paper or cardboard in grippers of the next cylinder. 


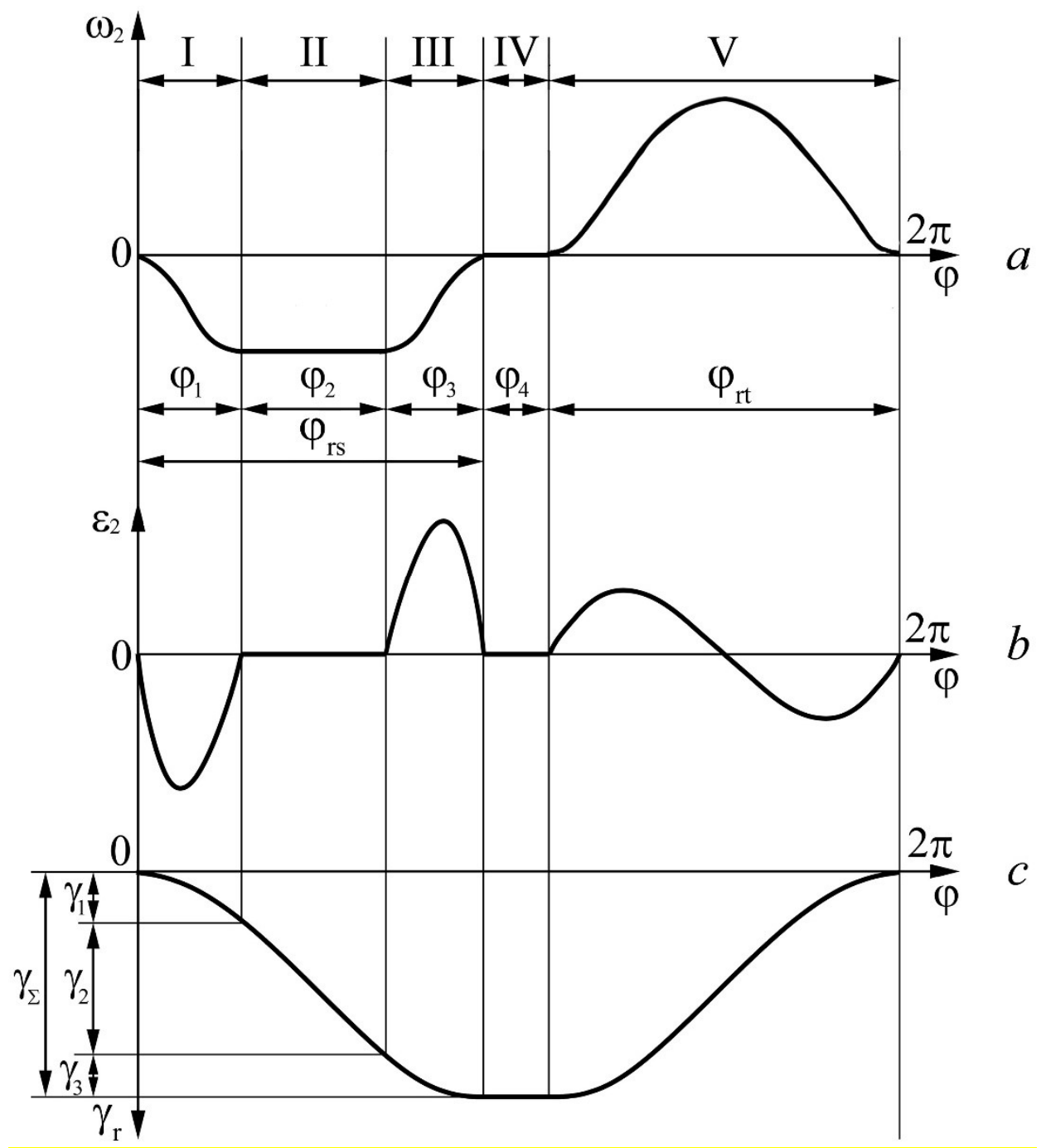

Figure 2. Diagram of change of angular velocities (a), acceleration (b) and displacement (c) of link 7 depending on the angle of rotation of the carrier 1

The phase angle of return of a follower will be equal to:

$$
\varphi_{\mathrm{rt}}=360^{\circ}-\left(\varphi_{1}+\varphi_{2}+\varphi_{3}+\varphi_{4}\right) .
$$

If there is no need for a constant velocity, then take $\varphi_{4}=0$, so then the phase angle of return of a follower is equal to:

$$
\varphi_{r t}=360^{\circ}-\left(\varphi_{1}+\varphi_{2}+\varphi_{3}\right) .
$$

The total swing angle of link 7 is equal to:

$$
\gamma_{\Sigma}=\gamma_{1}+\gamma_{2}+\gamma_{3} \text {. }
$$


Next, authors presents the determination method of the kinematics of the mechanism. The characteristic graphs of changes in the kinematic values of link 7 are show in fig. 2 a, b, c. A graph of changes in the angular velocity of the driven link 9 is in Fig. 2, d. The instant values of the kinematic factors of link 7 are determined for each section separately.

Section I - the run-up section of the rise period.

Angular displacement of the link 7:

$$
\gamma_{\mathrm{k}}=-2 \mathrm{a}_{\mathrm{k} 1} \cdot \gamma_{1}
$$

where $a_{k 1}$ is an invariant of instant displacements, $0 \leq a_{k 1} \leq 0,5$.

Angular velocity:

$$
\omega_{2}=-b_{k 1} \frac{\gamma_{1}}{\varphi_{1}} \cdot \omega_{1}
$$

where $b_{k 1}$ is an invariant of instant velocities, $0 \leq b_{k 1} \leq B_{1}$.

Angular acceleration:

$$
\varepsilon_{2}=-\mathrm{c}_{\mathrm{k} 1} \frac{\gamma_{1}}{\varphi_{1}^{2}} \cdot \omega_{1}^{2}
$$

where $c_{k 1}$ is an invariant of instant accelerations.

Section II - section of a constant speed.

Angular displacement:

$$
\gamma_{k}=-\gamma_{1}-\gamma_{2} \cdot k
$$

where $k$ is the relative time, $0 \leq k \leq 1$.

Angular velocity:

$$
\omega_{2}=-B_{1} \frac{\gamma_{1}}{\varphi_{1}} \cdot \omega_{1}=-\omega_{1}=\text { const }
$$

Angular acceleration $\varepsilon_{2}=0$.

Section III - the run-down section of the rise period.

Angular displacement: 


$$
\gamma_{k}=-\left(\gamma_{1}+\gamma_{2}-\gamma_{3}+2 a_{k 3} \cdot \gamma_{3}\right)
$$

where $a_{k 3}$ is an invariant of instant displacements in the section III, $0,5 \leq a_{k 3} \leq 1$.

Angular velocity:

$$
\omega_{2}=-b_{k 3} \frac{\gamma_{3}}{\varphi_{3}} \cdot \omega_{1}
$$

where $b_{k 3}$ is an invariant of instant velocities in the section III, $B_{3} \geq b_{k 3} \geq 0$.

Angular acceleration:

$$
\varepsilon_{2}=-c_{\mathrm{k} 3} \frac{\gamma_{3}}{\varphi_{3}^{2}} \cdot \omega_{1}^{2}
$$

Section IV-section of a dwell of the link 7.

The roller 5 is located on the maximum radius of the cam.

$$
\gamma_{\mathrm{k}}=-\gamma_{\Sigma}, \quad \omega_{2}=0, \quad \varepsilon_{2}=0 .
$$

In this section, the link 7 has no relative motion and the shaft 8 rotates at a constant speed $\omega_{1}$. The phase angle $\varphi_{4}$ can be set for technological reasons, for example, in the printing machine for a movement of the rotating stop-gripper at a constant speed during a transfer of the paper or cardboard sheet to the grippers of the cylinder. When there is no need for it, they accept $\varphi_{4}=0$.

Section $V$ - the return period.

Angular displacement:

$$
\gamma_{\mathrm{k}}=-\mathrm{a}_{\mathrm{k} 5} \cdot \gamma_{\Sigma}
$$

where $a_{k 5}$ is an invariant of instant displacements in the section V, $1 \geq a_{k 5} \geq 0$;

$\gamma_{\Sigma}=\gamma_{1}+\gamma_{2}+\gamma_{3}-$ the total swing angle of link 7 .

Angular velocity:

$$
\omega_{2}=b_{\mathrm{k} 5} \frac{\gamma_{\Sigma}}{\varphi_{\mathrm{rt}}} \cdot \omega_{1}
$$

where $b_{k 5}$ is an invariant of instant velocities in the section V; 
$\varphi_{\mathrm{rt}}$ is the phase angle of return of a follower.

Angular acceleration:

$$
\varepsilon_{2}=\mathrm{c}_{\mathrm{k} 5} \frac{\gamma_{\Sigma}}{\varphi_{\mathrm{rt}}^{2}} \cdot \omega_{1}^{2},
$$

where $c_{k 5}$ - invariant of instant accelerations.

Schemes of new planetary cam mechanisms offered to drive the slave links with periodic stops, where gears and transmission with levers are applied. Such mechanisms of constant structure makes it possible for the slave links to set a periodic stop for half or more of their cycle. Due to the smooth change of speed and acceleration of the driven link, the mechanisms provides trouble-free operation for a long time. The usage of levers in cam-lever mechanisms increases the accuracy of their operation. Planetary cam mechanisms can be used also in other branches, for example, with the aim to improve cardboard tray and case forming mechanisms [7]. 